\title{
EXAMPLE OF A SEQUENTIALLY INCOMPLETE REGULAR INDUCTIVE LIMIT OF BANACH SPACES
}

\author{
JAN KUCERA and KELLY MCKENNON \\ Department of Mathematics \\ Washington State University \\ Pullman, Washington 99164 \\ (Received August 22, 1989)
}

\begin{abstract}
A sequentially incomplete regular inductive limit of a sequence of Banach spaces is constructed.
\end{abstract}

KEY WORDS AND PHRASES. Regular locally convex inductive limit, sequentially complete locally convex space.

1980 MATHEMATICS SUBJECT CLASSIFICATION CODE (1985 Revision): Primary 46A12, secondary 46M40.

\section{INTRODUCTION.}

In [1] Jorge Mujica asks: Is every regular inductive limit of Banach spaces complete? A partial answer is in [2] with an example of a regular inductive limit which is quasi-incomplete. It is conjectured in [2] that the regular inductive limit might even be sequentially incomplete. Here we prove the conjecture. On the other hand, regular inductive limits of Banach spaces always have some completeness property, e.g. they are fast complete, see [3].

\section{MAIN RESULT.}

Let $N=\{1,2, \ldots\}$ and $R$ be the space of real numbers. For each $x=\left\{x_{i, j}\right\} \in R^{N \times N}$ and $n \in N$ we put $\|x\|_{i, n}=\max \left\{\frac{1}{i} \max \left\{\left|x_{i, j}\right| ; j<n\right\}, \sup \left\{\left|x_{i, j}\right| ; j \geq n\right\}\right\}$, $\|x\|_{n}=\sup \left\{\|x\|_{i, n} ; i \in N\right\}$, and $E_{n}=\left\{x \in R^{N \times N} ;\|x\|_{n}<\infty, \lim _{i \rightarrow \infty}\|x\|_{i, n}=0\right\}$.

For brevity, we also put $E_{n}(r)=\left\{x \in E_{n} ;\|x\|_{n} \leq r\right\}$.

LEMMA 1. The map $x \mapsto\|x\|_{n}: E_{n} \rightarrow R$ is a norm on $E_{n}$ and each functional $f_{i, j}: x \mapsto x_{i, j}:$ $E_{n} \rightarrow R$ is continuous.

PROOF. $\left|f_{i, j}(x)\right|=\left|x_{i, j}\right| \leq i\|x\|_{i, n} \leq i\|x\|_{n}$. 
LEMMA 2. Each $E_{n}$ is Banach.

PROOF. Let $\{x(k) ; k \in N\}$ be Cauchy. Then for each $i, j \in N$, the sequence $\left\{f_{i, j}(x(k)) ; k \in\right.$ $N\}$ is Cauchy in $R$ and thus converges to some $x_{i, j} \in R$. Put $x=\left\{x_{i, j}\right\}$. For any $i, k, n \in N$, we have $\lim _{m \rightarrow \infty}\|x(k)-x(m)\|_{i, n}=\|x(k)-x\|_{i, n}$, which implies $x \in E_{n}$ and $\lim _{k \rightarrow \infty}\|x(k)-x\|_{n}=0$.

LEMMA 3. The inductive limit $E=\underset{n \rightarrow \infty}{\text { ind }} \rightarrow E_{n}$ is regular.

PROOF. Let $B \subset E$ be not bounded in any $E_{n}$. Without a loss of generality we may assume that for any $n \in N$ there exists $x(n) \in B$ such that $\|x(n)\|_{n}>n$. This implies the existence of sequences $\{i(n)\},\{j(n)\} \subset N$ such that: either $j(n) \geq n$ and $\left|x(n)_{i(n), j(n)}\right|>n$ or $j(n)<n$ and $\left|x(n)_{i(n), j(n)}\right|>i(n) \cdot n$.

For each $k \in N$, choose $r_{k}>0$ so that $r_{k}^{-1}=\max \{i(n) ; n \leq k\}$ and denote by $V$ the convex hull of $\cup\left\{E_{k}\left(r_{k}\right) ; k \in N\right\}$. Tàke $k, n \in N, x=\left\{x_{i, j}\right\} \in E_{k}\left(r_{k}\right)$, and distinguish three cases:

(a) $j(n) \geq k$, which implies $\left|x_{i(n), j(n)}\right| \leq\|x\|_{k} \leq r_{k} \leq 1<\frac{1}{n}\left|x(n)_{i(n), j(n)}\right|$,

(b) $j(n)<k \& k \geq n$, which implies $\left|x_{i(n), j(n)}\right| \leq i(n) \cdot\|x\|_{k} \leq i(n) r_{k} \leq 1<$ $\frac{1}{n}\left|x(n)_{i(n), j(n)}\right|$,

(c) $j(n)<k \& k<n$, which implies $\left|x_{i(n), j(n)}\right| \leq i(n) \cdot\|x\|_{k} \leq i(n) r_{k} \leq i(n)<$ $\frac{1}{n}\left|x(n)_{i,(n), j(n)}\right|$.

For any case $\left|x_{i(n), j(n)}\right|<\frac{1}{n}\left|x(n)_{i(n), j(n)}\right|$. Since $x \in E_{k}\left(r_{k}\right), k \in N$, was arbitrary, the element $\frac{1}{n} x(n)$ cannot be expressed as a convex combination of elements from $\cup\left\{E_{k}\left(r_{k}\right) ; k \in\right.$ $N$ \}, i.e. $\frac{1}{n} x(n) \notin V$. Hence the 0 -neighborhood $V$ in $E$ does not absorb $B$ and $B$ is not bounded in $E$.

LEMMA 4. For each $i, j, n \in N$, put $x(n)_{i, j}=j^{-1}$ if $i \leq n, x(n)_{i, j}=0$ if $i>n$, and $x(n)=$ $\left\{x(n)_{i, j}\right\}$. Then:

(a) For each $n \in N, x(n) \in E_{1}(1)$.

(b) $\{x(n)\}$ is Cauchy in $E$.

(c) $\{x(n)\}$ does not converge in $E$.

\section{PROOF.}

(a) is evident.

(b) Let $V$ be a 0 -neighborhood in $E$. For each $n \in N$, choose $r_{n}>0$ so that $E_{n}\left(r_{n}\right) \subset V$. Further, choose $p, q \in N$ so that $p r_{1}>2$ and $q r_{p}>2$. For $m, n>q$, define $y, z \in E$ by:

$$
\begin{aligned}
& y_{i, j}=x(m)_{i, j}-x(n)_{i, j} \text { for } j \geq p, y_{i, j}=0 \text { otherwise } \\
& z_{i, j}=x(m)_{i, j}-x(n)_{i, j} \text { for } j<p, z_{i, j}=0 \text { otherwise. }
\end{aligned}
$$


Since

$$
\left|x(m)_{i, j}-x(n)_{i, j}\right| \leq\left\{\begin{array}{lll}
0 & \text { for } & i \leq q \\
\frac{1}{p} & \text { for } & i>q, j \geq p \\
1 & \text { for } & i>q, j<p
\end{array}\right\},
$$

We have $\|y\|_{1}=\sup \left\{\left|y_{i, j}\right|_{;} i \in N, j \geq p\right\} \leq \frac{1}{p}<\frac{1}{2} r_{1}$, and $\|z\|_{p}=\sup \left\{\left|\frac{1}{i} z_{i, j}\right| ; i>q\right.$, $j<p\}<\frac{1}{q}<\frac{1}{2} r_{p}$. Hence $y \in \frac{1}{2} E_{1}\left(r_{1}\right), z \in \frac{1}{2} E_{p}\left(r_{p}\right)$, and $x(m)-x(n)=y+z \in V$.

(c) Assume $x(n) \rightarrow x$ in $E$. Since each functional $f_{i, j}$, defined in Lemma 1 , is continuous on each $E_{n}$, it is also continuous on $E$. Then we have $x_{i, j}=f_{i, j}(x)=\lim _{n \rightarrow \infty} f_{i, j}(x(n))=$ $\lim _{n \rightarrow \infty} x(n)_{i, j}=j^{-1}$. Hence $\|x\|_{i, n}=\max \left(\frac{1}{i}, \frac{1}{n}\right)$ and $\lim _{i \rightarrow \infty}\|x\|_{i, n}=\frac{1}{n} \neq 0$, i.e. $x \notin E_{n}$ for any $n \in N$.

By combining Lemma to $1-4$, we get:

THEOREM. Regular inductive limit of Banach spaces may be sequentially incomplete.

\section{REFERENCES}

[1] MUJICA, J., Functional Analysis, Holomorphy and Approximation Theory II, North Holland 1984.

[2] KUCERA, J., MCKENNON, K., Completeness of regular inductive limits, Int. J. Math. \& Math Sci. Vol. 12, No. 3 (1989), 425-428.

[3] DEWILDE, M., Closed Graph Theorems and Webbed Spaces, Pitman, London 1978. 


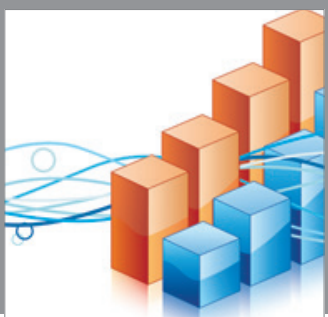

Advances in

Operations Research

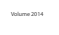

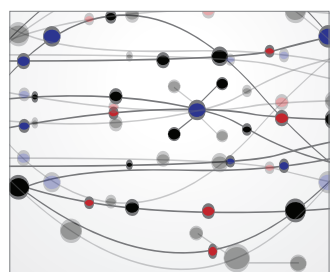

\section{The Scientific} World Journal
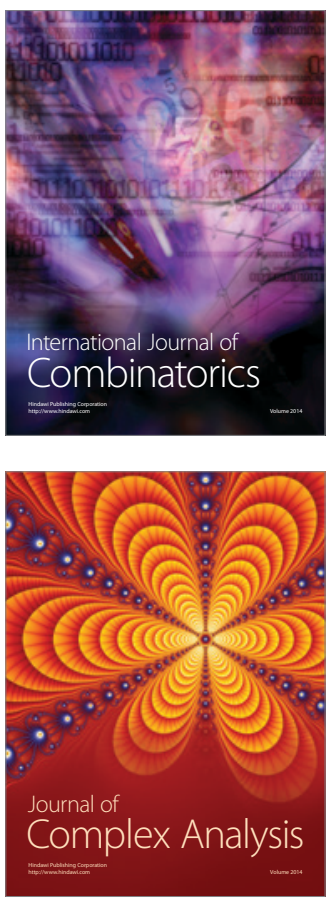

International Journal of

Mathematics and

Mathematical

Sciences
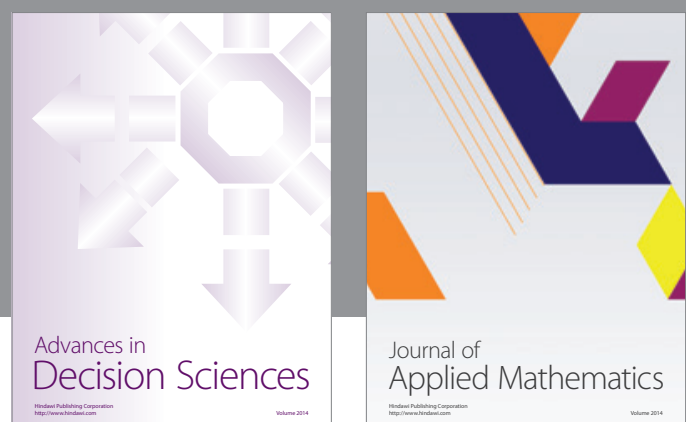

Journal of

Applied Mathematics
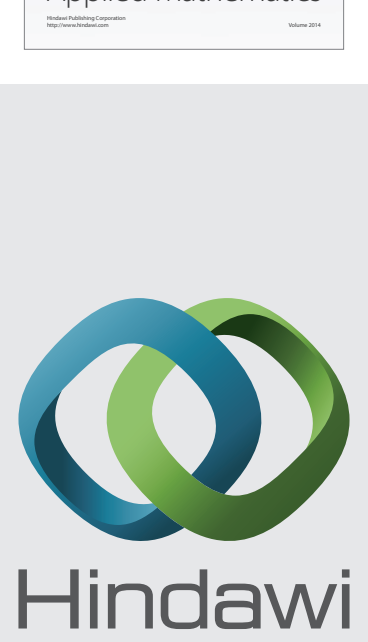

Submit your manuscripts at http://www.hindawi.com
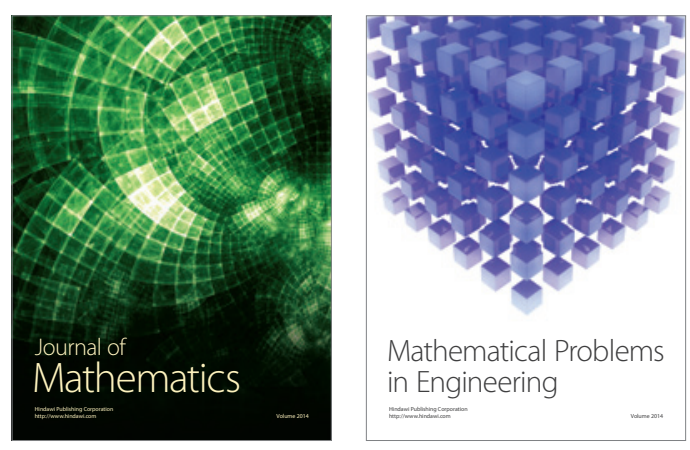

Mathematical Problems in Engineering
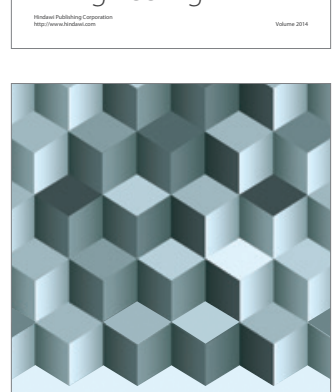

Journal of

Function Spaces
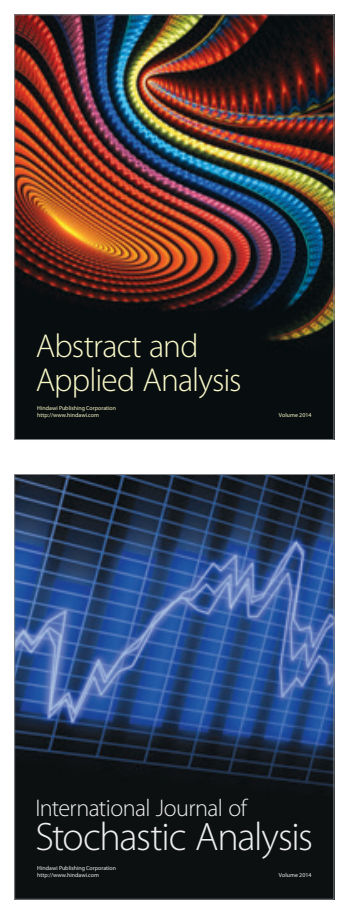

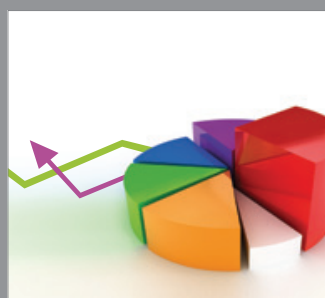

ournal of

Probability and Statistics

Promensencen
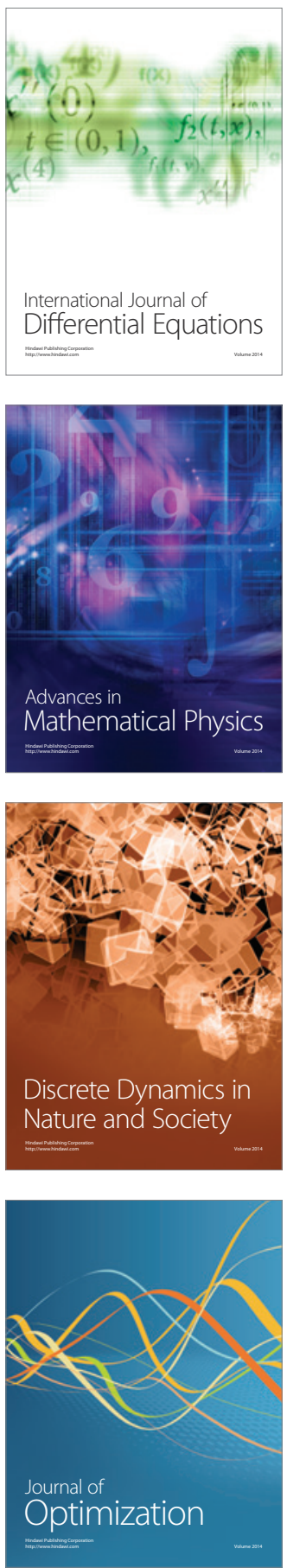